\title{
Evaluación de la eficacia y seguridad del cerclaje cervical temporal tras una adenomectomía prostática
}

\author{
MaJ. Cancho Gil, R. Díz Rodríguez, M. Virseda Chamorro, C. Alpuente Román, \\ J.A. Cabrera Cabrera, P. Paños Lozano
}

Servicio de Urología. Hospital Central de la Defensa. Madrid.

Actas Urol Esp 2006; 30 (1): 53-56

\section{RESUMEN}

EVALUACIÓN DE LA EFICACIA Y SEGURIDAD DEL CERCLAJE CERVICAL TEMPORAL TRAS UNA ADENOMECTOMÍA PROSTÁTICA

Introducción: El cerclaje del cuello vesical disminuye el riesgo de sangrado tras adenomectomía prostática, pero aumenta el riesgo de padecer esclerosis cervical postoperatoria. Nuestro objetivo es valorar la eficacia y seguridad terapéutica del cerclaje temporal del cuello vesical tras adenomectomía prostática.

Material y métodos: Se realizó un estudio longitudinal perspectivo en una cohorte de 25 pacientes de edad media 68,5 años (desviación típica, 2,6 años), sometidos adenomectomía prostática transvesical tipo Freyer con cerclaje cervical temporal utilizando polipropileno del número 1 que se retiró a las 24 horas de la cirugía. Para valorar la utilidad hemostática del cerclaje se midió el descenso del hematocrito y la concentración de hemoglobina a las 24 horas de la intervención. Para evaluar la aparición de secuelas obstructivas se midió el flujo miccional máximo a los tres meses de la cirugía.

Resultados: Se observó una disminución media del valor hematocrito a las 24 horas de la cirugía del 7,3\%, y una disminución media de la hemoglobina a las 24 horas de la cirugía de 2,7 gr./dl. En ninguno de los casos la concentración de hemoglobina postoperatoria fue inferior a $8 \mathrm{mg} / \mathrm{dl}$, por lo que no se precisó transfusión. Los resultados obtenidos en la medición de la flujometría realizada a los 3 meses de esta mostró que 24 de los 25 pacientes intervenidos presentaron a los 3 meses un flujo máximo superior a $15 \mathrm{ml} / \mathrm{sg}$.

Conclusiones: El cerclaje vesical temporal es una buena técnica hemostática. La retirada precoz del cerclaje evita las secuelas tardías (esclerosis de cuello vesical) que origina el cerclaje permanente.

Palabras clave: Prostatectomía. Sangrado. Cerclaje cervical. Esclerosis de cuello.

\section{ABSTRACT}

ASSESMENT OF EFFECTIVENESS AND SECURITY OF BLADDER NECK CERCLAGE AFTER TRANSVESICAL PROSTATECTOMY

Introduction: The Bladder neck cerclage diminishes the risk of bledding after transvesical prostatectomy, but it increases the risk of suffering postoperative bladder neck sclerosis. Our objective is to value the effectiveness and therapeutic security of the temporary bladder neck cerclage after transvesical prostaectomy.

Material and methods: It was carried out a prospective and longitudinal study in a cohort of 25 age patients mean age 68,5 years (standard deviation, 2,6 years), subjected to transvesical prostatectomy (Freyer) with bladder neck cerclage using polipropilene number 1 suture, that was retired at the 24 hours of the surgery. To value the hemostatic utility of bladder neck cerclage, it was measured the hematocrit and hemoglobin concetration at the 24 hours of the intervention. To evaluate the appearance of obstructive sequels, the maximun urinary flow was measured three months after the surgery.

Results: It was observed a mean hematocrit at the 24 hours of surgery of 7,3\%, and a mean decrease of the hemoglobin at the 24 hours of the surgery of $2,7 \mathrm{gr} / \mathrm{dl}$. In any cases the postoperative hemoglobin concentration was inferior to $8 \mathrm{mg} / \mathrm{dl}$, therefore, it was not necessary transfusion. The uroflowmetry carried out at $3 \mathrm{months}$ of surgery showed that 24 of the 25 intervened patients presented a maximum flow superior to $15 \mathrm{ml} / \mathrm{sg}$.

Conclusions: The temporary bladder neck cerclage is a good hemostatic technique. The precocious cercalge retreat avoids the late sequels (bladder neck sclerosos), that originates the permanent cerclage.

Keywords: Prostatectomy. Bledding. Bladder neck cerclage. Bladder neck sclerosis. 
$\mathrm{D}^{2}$ esde que en 1900 Freyer realizara su primera adenomectomía prostática transvesical han sido innumerables las técnicas descritas encaminadas a disminuir el sangrado postquirúrgico, una de las complicaciones inmediatas más importantes y graves de la esta cirugía. Actualmente la mayoría de los varones que requieren una operación prostática, son sometidos a resección transuretral. Sin embargo, aproximadamente un $10 \%$ de estos pacientes requieren una enucleación abierta del adenoma ${ }^{1}$.

El cerclaje cervical permanente, al colapsar la vascularización prostática localizada principalmente a nivel cervical, ha demostrado su utilidad para evitar la hemorragia postquirúrgica ${ }^{2,3}$. Sin embargo, la cirugía sobre el cuello vesical, aumenta el riesgo de secuelas tardías como las esclerosis de cuello vesical ${ }^{4}$.

El cerclaje cervical temporal evitaría el sangrado post-quirúrgico inmediato a la vez que al solo permanecer 24 horas no aumentaría significativamente el riesgo de esclerosis de cuello vesical tardías. El objetivo que nos propusimos en este trabajo fue valorar la seguridad, eficacia hemostática y la ausencia de secuelas tardías del cerclaje cervical temporal tras una adenomectomía prostática transvesical tipo Freyer.

\section{MATERIAL Y MÉTODOS}

Se realizó un estudio longitudinal prospectivo en una cohorte de 25 pacientes de edad media 68,5 años (desviación típica, 2,6 años), que iban a ser sometidos a una adenomectomía por hiperplasia prostática benigna. Los criterios de inclusión y exclusión en el estudio se muestran en las Tablas 1 y 2 respectivamente.

A cada paciente, previamente a su inclusión en este estudio se le realizaron las siguientes determinaciones: Historia clínica detallada con

Tabla 1

Criterios de inclusión en el estudio

- Edad comprendidas entre 65 y 80 años.

- Próstata ecográficamente superior a 70cc.

- Flujometría con un flujo miccional máximo no superior a $10 \mathrm{ml} / \mathrm{sg}$

- IPSS no inferior a 8 .

- Analísis de sangre, orina y coagulación normales.

- PSA inferior a $4 \mathrm{ng} / \mathrm{ml}$.
Tabla 2

Criterios de exclusión en el estudio

- Pacientes con enfermedades sistémicas (diabetes, lupus, etc.).

- Pacientes con enfermedades cardíacas que le obliguen a tratamientos con antiagregantes o anticoagulantes.

- Pacientes sometidos con anterioridad a cirugías en el área pelviana.

- Pacientes con infecciones urinarias de repetición.

- Pacientes con tratamientos previos por HBP.

- Pacientes que previamente hallan portado sonda vesical.

valoración del IPSS, hemograma previo a la cirugía y otro a las 24 horas tras la intervención, bioquímica con iones y estudio de coagulación, Analítica de orina con cultivo, estudio de coagulación, preoperatorio cardiológico, ecografía urológica con medición de residuo postmiccional, flujometría previa a la cirugía y otra a los tres meses.

La técnica quirúrgica se realizó de la siguiente manera: todos los pacientes fueron anestesiados con una anestesia raquídea. Se les realizó una adenomectomía prostática transvesical tipo Freyer con cerclaje cervical temporal (Figs. 1 y 2) utilizando polipropileno del número 1 que se retiró a las 24 horas de la cirugía.

Durante el postoperatorio se siguió el siguiente protocolo: a todos los pacientes se les mantuvo con 2.000 cc de sueroterapia con glucosado al 5\% y fisiológico alternos durante las primeras 24 horas. Como analgesia se usó una ampolla de ketocoralaco cada 8 horas y como antibioterapia se utilizó un gramo de amoxicilina con clavulónico cada 8 horas. Todos los pacientes mantuvieron un suero

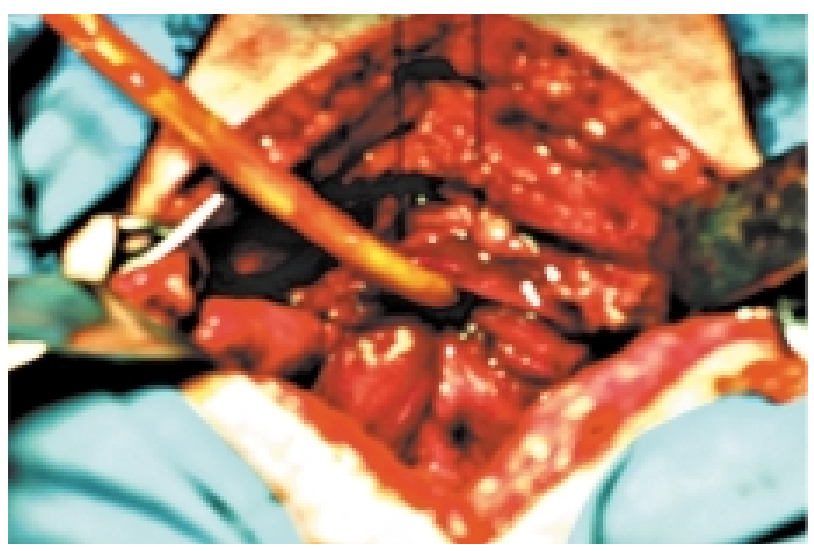

FIGURA 1.- Cerclaje cervical postadenomectomia prostática con polipropileno del número 1 . 


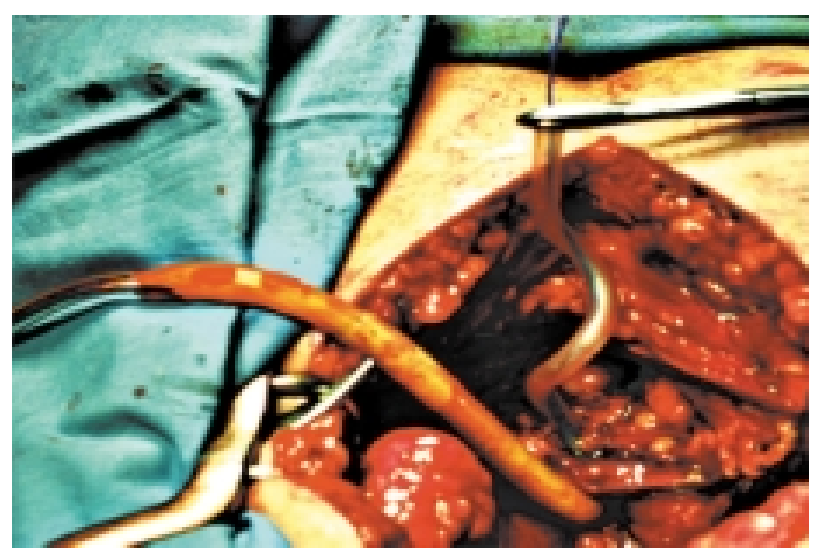

FIGURA 2.- Ajuste del cerclaje con un redón del número 14.

lavador continuo durante el postoperatorio. A todos los pacientes se le realizaría una analítica de sangre en la mañana del día siguiente de la cirugia.

Para valorar la eficacia hemostática de esta técnica y comprobar la ausencia de esclerosis de cuello vesical secundarias se utilizó como variable el valor hematocrito y la hemoglobina a las 24 horas de la cirugía. Para valorar la presencia de patología obstructiva se realizó una flujometría tres meses después de ser intervenidos.

Para el análisis estadístico de los datos obtenidos se utilizó el programa SPSS-PC de la universidad de Washington. Previo al estudio estadístico se evaluó el ajuste de la muestra a una distribución normal, según el test de Kolmogorov-Smirnov. Una vez confirmada que la muestra se ajusta a una distribución normal se efectua el análisis de los datos según test paramétricos consistentes en la comparación de las medias para muestras pareadas. El nivel mínimo de significación se fijó en $\mathrm{p}<0,05$ bilateral.

\section{RESULTADOS}

La distribución de los resultados obtenidos en la medición de las medias del valor hematocrito previos a la cirugía y a las 24 horas se muestra en la Tabla 3. Se observó una disminución media del valor hematocrito a las 24 horas de la cirugía del 7,3\%. Esta diferencia fue significativa.

La distribución de los resultados obtenidos en la medición de las medias de la hemoglobina previos a la cirugía y a las 24 horas se muestra en la Tabla 4. Se observó una disminución media de la hemoglobina a las 24 horas de la cirugía de $2,7 \mathrm{~g} / \mathrm{dl}$. Esta diferencia fue significativa.

Ningún paciente precisó transfusión sanguínea postquirúrgica y que como única complicación en el postoperatorio inmediato se presentó un seroma.

Los resultados obtenidos en la medición de la flujometría realizada a los 3 meses de ésta mostró que 24 de los 25 pacientes intervenidos presentaron a los 3 meses un flujo máximo superior a $15 \mathrm{ml} / \mathrm{sg}$.

\section{DISCUSIÓN}

En nuestro estudio se observó un descenso significativo tanto de la concentración de hemoglobina, como del hematocrito a las 24 horas de la cirugía. Sin embargo, en ninguno de los casos este descenso fue lo suficientemente intenso como

\section{Tabla 3}

Variación de los valores de hematocrito

\begin{tabular}{ccccccc}
\hline $\begin{array}{c}\text { Número de } \\
\text { pacientes }\end{array}$ & $\begin{array}{c}\text { Hto medio } \\
\text { previo }\end{array}$ & $\begin{array}{c}\text { Hto medio } \\
\text { postQ }\end{array}$ & $\begin{array}{c}\text { Diferencia } \\
\text { media }\end{array}$ & $\begin{array}{c}\text { Diferencia } \\
\text { mayor }\end{array}$ & $\begin{array}{c}\text { Diferencia } \\
\text { menor }\end{array}$ & $\begin{array}{c}\text { Desviación } \\
\text { típica }\end{array}$ \\
\hline 25 & $41,3 \%$ & $34 \%$ & $7,3 \%$ & $9,1 \%$ & $6,2 \%$ & 2,10 \\
\hline
\end{tabular}

\section{Tabla 4}

Variación de los valores de hemoglobina

\begin{tabular}{ccccccc}
\hline Pacientes & $\begin{array}{c}\text { Hb media } \\
\text { previa }\end{array}$ & $\begin{array}{c}\text { Hb media } \\
\text { postQ }\end{array}$ & $\begin{array}{c}\text { Diferencia } \\
\text { media }\end{array}$ & $\begin{array}{c}\text { Diferencia } \\
\text { mayor }\end{array}$ & $\begin{array}{c}\text { Diferencia } \\
\text { menor }\end{array}$ & $\begin{array}{c}\text { Desviación } \\
\text { típica }\end{array}$ \\
\hline 25 & $14,1 \mathrm{~g} / \mathrm{dl}$ & $11,4 \mathrm{~g} / \mathrm{dl}$ & $2,7 \mathrm{~g} / \mathrm{dl}$ & $4,2 \mathrm{~g} / \mathrm{dl}$ & $2,3 \mathrm{~g} / \mathrm{dl}$ & 1,09 \\
\hline
\end{tabular}


para requerir transfusión sanguínea. En general, se considera que se precisa transfusión cuando concentración postquirúrgica de hemoglobina inferior o igual a $8,0 \mathrm{~g} / \mathrm{dl}^{5,6}$. Ninguno de nuestros pacientes sufrió un descenso de la concentración de hemoglobina inferior o igual a esta cifra. El valor más bajo de concentración de hemoglobina postoperatorio fue de $9,8 \mathrm{~g} / \mathrm{dl}$.

$\mathrm{El}$ porcentaje de pacientes transfundidos en el caso de cirugía prostática simple, varía según la técnica empleada y las series publicadas. Stephenson et al. ${ }^{7}$, registran un $14 \%$ de transfusiones entre los pacientes intervenidos de cirugía prostática. Serretta et al. $^{8}$ en una serie 1.804 pacientes intervenidos mediante prostatectomía abierta refieren un $12 \%$ de hemorragias severas y un porcentaje de pacientes que requirieron transfusión sanguínea del 8\%. La hemostasia cuidadosa no parece disminuir el riesgo de transfusión, así Shaheen y Quinlan ${ }^{9}$, refieren un descenso medio de $2,2 \mathrm{~g} / \mathrm{dl}$ de la concentración de hemoglobina, con un $16 \%$ de transfusiones en pacientes sometidos a prostatectomía abierta con control exhaustivo de la hemostasia. Únicamente Hill y Njoroge ${ }^{10}$, publican una incidencia de transfusiones postoperatorias en cirugía prostática abierta menor del 5\%. La resección transuretral de próstata convencional con control cuidadoso de la hemostasia también estaría expuesta a este tipo de complicaciones, aunque en una proporción de alrededor del $3 \%$ de los $\operatorname{casos}^{11}$.

La esclerosis del cuello vesical se ve favorecida por una cirugía agresiva sobre esa estructura $\mathrm{y}$ por procesos inflamatorios crónicos ${ }^{12}$. Por eso, algunos autores también han postulado realizar un cerclaje temporal del cuello vesical ${ }^{13}$.

La esclerosis de cuello vesical produce obstrucción del tracto urinario y como consecuencia un descenso del flujo miccional ${ }^{14}$. Aunque el único método diagnóstico fiable para comprobar la presencia de obstrucción urinaria, son los estudios urodinámicos de presión del detrusor y flujo miccional, la persistencia de un flujo miccional disminuido puede ser compatible con la existencia de esa alteración miccional ${ }^{15}$. En nuestro estudio, excepto en un caso, todos los pacientes presentaron un flujo miccional máximo a los tres meses de la cirugía superior o igual a $15 \mathrm{ml} / \mathrm{s}$, valor que hace muy improbable la existencia de una obstrucción del tracto urinario.
En conclusión, podemos indicar que:

1. El cerclaje cervical temporal tras una adenomectomía prostática tipo Freyer es una buena técnica hemostática.

2. La retirada precoz del cerclaje evita las secuelas tardías (esclerosis de cuello vesical) que origina el cerclaje permanente.

3. El uso de polipropileno monofilamento para el cerclaje cervical temporal permite:

El cierre hermético del cuello vesical y por tanto evita el sangrado.

Se puede retirar con facilidad por su fácil deslizamiento.

\section{REFERENCIAS}

1. Van Arsdalen K. Prostate surgery. Clinics in Geriatric Medicine 1990;6(3):609-631.

2. Amen-Palma JA, Bernardo R. Hemostatic technique: extracapsular prostatic adenomectomy. J Urol 2001;166(4):1364-1367.

3. Van Glabeke E, Corsia G, Barrou B, Conort P, Bitker MO, Richard F Anal. Évaluation du risk d'hemorragie dans la prostatectomie transvesicale avec cerclage du col vessical. Prog Urol 2000;10(6): 1177-1183.

4. Moudouni SM, Nouri M, Lrhorfi MH, Koutani A, Iben Attya Andaloussi A, Hachimi M, Lakrissa A. Esclérosè secundaire du col vessical aprés trataiment chirurgicale de l'hypertorphie benigne de la prostate. Ann Urol (Paris) 1999;33(4):252-255.

5. Lum G. Should the transfusion trigger and hemoglobin low critical limit be identical? Ann Clin Lab Sci 1997;27(2):130-134.

6. Carson JL, Duff A, Berlin JA, Lawrence VA, Poses RM, Huber EC, O'Hara DA, Noveck H, Strom BL. Perioperative blood transfusion and postoperative mortality. JAMA 1998;279(3):199-205.

7. Stephenson WP, Chute CG, Guess HA, Schwartz S, Lieber M. Incidence and outcome of surgery for benign prostatic hyperplasia among residents of Rochester, Minnesota: 1980-87. A population-based study. Urology 1991;38(1 Suppl):32-42.

8. Serretta V, Morgia G, Fondacaro L, Curto G, Lo bianco A, Pirritano D, Melloni D, Orestano F, Motta M, Pavone-Macaluso M; Members of the Sicilian-Calabrian Society of Urology. Open prostatectomy for benign prostatic enlargement in southern Europe in the late 1990s: a contemporary series of 1800 interventions. Urology 2002;60(4):623-627.

9. Shaheen A, Quinlan D. Feasibility of open simple prostatectomy with early vascular control. BJU Int 2004;93(3):349-352.

10. Hill AG, Njoroge P. Suprapubic transvesical prostatectomy in a rural Kenyan hospital. East Afr Med J 2002;79(2):65-67.

11. Berger AP, Wirtenberger W, Bektic J, Steiner H, Spranger R, Bartsch G, HorningerW. Safer transurethral resection of the prostate: coagulating intermittent cutting reduces hemostatic complications. J Urol 2004;172(2):780

12. Perez Aebej JA, Cameo Rico MI, Valdivia Uria JG, Espuela Orgaz R. Escvlerosis del cuello vesical postoperatoria: mecaniosmos patogénicos. Arch Esp Urol 1991;44(10):1167-1176.

13. Condie JD Jr, Cutherell L, Mian A. Suprapubic prostatectomy for benign prostatic hyperplasia in rural Asia: 200 consecutive cases. Urology 1999;54(6):1012-1016.

14. Gomha MA, Boone TB. Voiding patterns in patients with post-prostatectomy incontinence: urodynamic and demographic analysis. J Uro 2003;169(5):1766-1769.

15. Chancelor M, Blaivas J, Kaplan S, Axelrod S. Bladder outlet obstruction versus impaired detrusor contraction J Urol 1991;145:810-813.

Dr. M. Virseda Chamorro

(Trabajo recibido el 24 de mayo 2005) 